\title{
PERCEPCIONES DE LA INSTRUCCIÓN BASADA EN LOS PRINCIPIOS DE UN ENFOQUE LÉXICO EN EL AULA DE LENGUA EXTRANJERA
}

\section{PERCEPTIONS OF FOREIGN LANGUAGE INSTRUCTION BASED ON A LEXICAL APPROACH}

\author{
Mercedes Pérez Serrano \\ Universidad Nebrija \\ mperezse@nebrija.es
}

Enviado: $19 / 09 / 2018$

Aceptado: 06/09/2019

\section{Resumen}

Se sabe que la lengua formulaica plantea problemas a los aprendices de lenguas extranjeras. Sobre esta cuestión se ha venido llamando la atención desde el primer Enfoque Léxico (Lewis 1993). En este estudio, ponemos a prueba algunas de las recomendaciones de este enfoque y sus desarrollos posteriores para indagar acerca de la valoración y las percepciones de estudiantes y profesora. Para ello, dividimos a los estudiantes en dos grupos, el grupo de captación, en el que se implementó la rutina de captar los bloques léxicos en el input, y el grupo de actividades explícitas, cuyos integrantes, después de exponerse al input, hicieron actividades para trabajar las unidades léxicas meta. Después de ocho semanas de tratamiento, se administró un cuestionario de respuestas abiertas. Asimismo, la profesora escribió periódicamente un diario en el que recogió sus impresiones. El análisis de datos da pistas sobre decisiones metodológicas para el futuro.

\begin{abstract}
It is widely accepted that formulaic language is problematic for foreign language learners. This topic has attracted researchers' attention since the principles of the first Lexical Approach were published (Lewis 1993). In this study, some of the recommendations of this approach and its developments are put to the test in order to gauge the students' and instructor's perceptions and attitudes. To that end, we implemented different versions of a lexical approach. Students were randomly assigned to either the noticing group -students' attention was raised to chunks in the text and they were invited to notice them- or the explicit activities group -after being exposed to the same input, students did activities targeting the studied lexical units. After the eight-week treatment, an open-ended questionnaire was administered. The instructor kept a journal in which she collected her impressions on the implementation of a lexical approach. These evaluations shed some light on future methodological decisions.
\end{abstract}

Para citar este artículo / To cite this article: Pérez Serrano, Mercedes (2019). Percepciones de la instrucción basada en los principios de un enfoque léxico en el aula de lengua extranjera. ELUA, 33: 157-172. doi: 10.14198/ELUA2019.33.8

Enlace / Link: http://dx.doi.org/10.14198/ELUA2019.33.8 
PALABRAS CLAVE: Léxico, lengua extranjera, bloques léxicos, actitudes, valoración.
KEYWORDS: Vocabulary, foreign language, lexical chunks, attitudes, evaluation.

\section{INTRODUCCIÓN}

Han pasado ya más de dos décadas desde la publicación de The lexical approach (Lewis 1993) y sus publicaciones subsiguientes (Lewis 1997, 2000) en las que detalla los principios del llamado Enfoque Léxico (EL en adelante). En estos trabajos, Lewis y sus colaboradores sientan las bases de un nuevo enfoque cuya mayor contribución es el reconocimiento del componente léxico (Lewis, 1993: 31), sin salirse de los presupuestos del Enfoque Comunicativo.

Los principios esenciales en los que se asienta este primer enfoque léxico son los siguientes: (1) la lengua es léxico gramaticalizado y el discurso está prefabricado de acuerdo con el principio de idiomaticidad (Sinclair 1991) y (2) el vocabulario no consiste únicamente en palabras sino en unidades superiores llamados bloques léxicos (ing. lexical chunks), entre los que se encuentran las colocaciones léxicas. Se sabe que las colocaciones (dar un paseo, tomar el sol) ofrecen especial dificultad a los aprendices de lengua extranjera (Nekrasova 2009; Li y Schmitt 2010; Yamashita y Jiang 2010). En torno a estas ideas se desarrolla una metodología de enseñanza de inglés como lengua extranjera en la que el entrenamiento para la captación de las unidades léxicas en el input y el aprendizaje incidental durante actividades de lectura y/o escucha tienen una gran importancia. Esta es la razón por la cual se ha señalado la captación de bloques (ing. chunk noticing) como el centro de las prácticas pedagógicas propuestas por Lewis y sus colaboradores (Boers y Lindstromberg 2009): exponer al aprendiente a grandes cantidades de input y entrenarlo para la captación de los bloques que allí aparecen.

Una de las críticas más repetidas en torno al enfoque léxico es la falta de aportación de evidencia empírica. De acuerdo con Schmitt y Alali (2012), se han dado muchos consejos pedagógicos, se ha propuesto implementar determinadas rutinas y llevar a cabo diversas actividades para enseñar-aprender bloques léxicos y sin embargo, se ofrecen pocas pruebas fehacientes o datos sobre cómo se adquieren y se incorporan al propio discurso estas unidades lingüísticas. Estos autores exceptúan a Boers y otros autores de su círculo a quienes se refiere como "unos de los pocos académicos que han investigado sobre la eficacia de las técnicas pedagógicas para enseñar fórmulas" (Schmitt y Alali 2012: 156) (traducción propia). Así, en los últimos años, se han publicado numerosos estudios que ponen a prueba los principios del enfoque léxico (vid. Boers et al. 2006; Stengers 2009) y que tratan de desarrollar los métodos de instrucción más eficientes (Boers et al. 2014; Stengers y Boers 2015; Boers et al. 2016b, por nombrar algunos).

En cualquier caso, el interés investigador por el papel del léxico en las dos últimas décadas es innegable. Desde aquellos primeros estadios del enfoque léxico, se han sucedido numerosas investigaciones acerca de cómo se adquiere el léxico y cuáles son los métodos de instrucción más eficaces. Es por ello que, siguiendo a Boers y Lindstromberg (2009), utilizaremos la expresión un enfoque léxico en lugar de el enfoque léxico para no identificarlo únicamente con las propuestas de Lewis (1993) sino con todas aquellas propuestas que tienen en cuenta la dimensión sintagmática del léxico (es decir, que no contemplan el léxico 
como una lista de palabras individuales sino que tienen en cuenta la combinatoria, poniendo énfasis en la enseñanza aprendizaje otras unidades léxicas más amplias como las fórmulas, las colocaciones, las locuciones, etc.) y que se han ido sumando a lo largo de los años.

Por otra parte, a pesar de este creciente interés en los procesos que subyacen a la adquisición y el aprendizaje de colocaciones y otros bloques léxicos, es nuestra impresión que, salvo omisión involuntaria, existe de momento poca investigación acerca de las percepciones, actitudes y estrategias de aquellos implicados en el proceso de enseñanza-aprendizaje, a saber, aprendiz y profesor, ante la metodología empleada y las actividades usadas para promover el desarrollo de la competencia colocacional. La mayoría de estudios que desde la lingüística aplicada se centran en el aprendizaje o la adquisición de la combinatoria léxica y que ponen a prueba los distintos presupuestos de un enfoque léxico son de corte cuantitativo, lo cual nos ofrece una visión muy esclarecedora sobre qué prácticas pedagógicas son más eficientes de cara al aprendizaje pero no nos permite apreciar las percepciones de aprendices y profesores ante dichas prácticas.

Con este estudio se pretende dar voz a estudiantes y profesor, tratando de ofrecer pistas para llegar a una mejor comprensión de las percepciones, actitudes y dificultades que encontraron aprendientes y profesores ante la implementación de un enfoque léxico durante 8 semanas de un curso general de español como lengua extranjera.

\section{LOS BLOQUES LÉXICOS Y SU TRATAMIENTO EN EL AULA DE LENGUAS EXTRANJERAS}

Los bloques léxicos se han definido como "secuencias de palabras que los hablantes nativos sienten como naturales y que constituyen la formulación preferida para expresar una idea o propósito" (Lindstromberg y Boers 2008: 7). Así, se trata de una categoría poco restrictiva desde el punto de vista formal o funcional pero operativa para el aula de lenguas extranjeras y que engloba elementos tan dispares como colocaciones (tomar una decisión), locuciones (meter la pata) o marcadores discursivos (de todas formas). Dada la gran cantidad de bibliografía dedicada a la delimitación y la clasificación de las unidades léxicas complejas (vid. Wray 2002), en este estudio no entraremos en discusión acerca de dicha delimitación y tomaremos, para la elección de las unidades meta, la definición antes citada de Lindstromberg y Boers (2008).

Se ha señalado que la falta de dominio de los bloques léxicos o de competencia colocacional en aprendices de nivel intermedio -nivel en el que se realiza el presente estudioocasiona ciertos problemas que impiden al aprendiz despegar desde ese nivel intermedio hasta niveles de dominio más altos. Algunos de estos los recogen Ying y O’Neill (2009):

- los aprendices, al no tener al alcance de la mano el bloque léxico adecuado, dan rodeos que les llevan a cometer otro tipo de errores (Lewis 2000);

- las producciones lingüísticas que no incluyen este tipo de unidades léxicas suenan “extrañas" o "no nativas" (Yang y Hendicks 2004);

- uso excesivo de términos generales que dan lugar a un estilo simplificado y "poco interesante" (Singleton 1999, citado en Ying y O’Neil 2009).

Además, se sabe que las colocaciones ofrecen una mayor dificultad que las palabras individuales porque, al estar formadas por palabras a medio conocer, no llaman la atención por sí mismas en el input. Así, un aprendiz anglófono que se expone a la colocación tomar una de- 
cisión puede no reparar en la elección del verbo tomar -porque le resulta conocido y no ofrece dificultades de comprensión- y es probable que a la hora de producir dicha colocación lo haga como *hacer una decisión, por interferencia de su lengua materna (en la que esta colocación se forma con el verbo make, to make a decision). Además, Boers y Lindstromberg (2009) aducen otra serie de razones que dificultan su aprendizaje frente al de palabras individuales como su frecuente carácter idiomático, la ausencia de espacios que las delimiten como ocurre con las palabras o la posible discontinuidad en el discurso, como en di un larguísimo paseo, donde sería más complicado de percibir que dar un paseo constituye una unidad léxica.

Todos estos problemas han hecho que tanto profesores como creadores de materiales se den cuenta de la necesidad de llamar la atención de los aprendices sobre los bloques léxicos y que los investigadores exploren cuál es la forma más eficiente para que se produzca su adquisición. Desde un punto de vista cuantitativo, parece haber acuerdo en que la instrucción explícita es más eficaz que otras técnicas pedagógicas como la captación en el input a través de distintas técnicas de realce -negrita, subrayados, preguntas del profesor, etc.- (Le-Thi, Rodgers y Pellicer 2018; Pérez Serrano 2018).

Asimismo, hemos recogido en la bibliografía algunos estudios de corte cualitativo que, aunque con premisas y objetivos diferentes al nuestro, exploran también en el desarrollo de la competencia colocacional desde una perspectiva introspectiva del estudiante. En Barfield (2006) se explora cómo los aprendientes organizan, comprenden e interpretan su conocimiento léxico y colocacional a lo largo del tiempo. En otro estudio, Yang y Hendricks (2004) examinan cómo usaron los estudiantes el llamado Collocation Awareness- raising approach o el método de concienciación de las colocaciones para el aprendizaje de las mismas de cara a tareas de escritura. Asimismo, Ying y O’Neill (2009) estudian cómo manejan los estudiantes el aprendizaje de las colocaciones a través del denominado enfoque 'AWARE'. Sin embargo, consideramos que, para conocer cuál es la mejor manera de aplicar los principios del enfoque léxico en el aula de ELE, es importante indagar acerca de las actitudes y las reacciones de los aprendices y profesores ante las distintas prácticas pedagógicas que propone este enfoque. Nuestro estudio tiene como objetivo cubrir este hueco en la literatura, indagando acerca de cómo valoran los estudiantes dos de esas prácticas pedagógicas (realización actividades explícitas destinadas al aprendizaje de las unidades meta y captación de los bloques léxicos en el input), así como explorando las dificultades a las que se enfrenta el profesor ante una y otra. Es decir, recoger y analizar la valoración de una instrucción basada en los principios de un enfoque léxico por parte de aprendices y profesor.

\section{METODOLOGÍA}

\subsection{Participantes}

Para este estudio se contó con 36 estudiantes de español como lengua extranjera matriculados en el curso SPAN1201 de la Universidad de Columbia y Barnard college (EE.UU.) y cuya lengua materna es el inglés. Todos ellos son adultos con edades comprendidas entre

1 AWARE es un acrónimo que significa: Awareness raising of language features, in particular collocations [...]; Why should we learn collocations?, helping learners see the rationale for/meaning of learning what they learn; Acquiring noticed collocations using various strategies [...]; Reflection of learning process and content; Exhibiting what has been learnt (Ying y O’Neill 2009, 183). 
los 18 y los 25 años que están estudiando un grado en la universidad. El 69\% son mujeres.

Su nivel de español se corresponde aproximadamente con un B1 del MCER. Se les ha asignado al curso Intermedio I (SPAN1201) o bien porque han superado el curso anterior o bien porque han hecho un examen de nivel que los ha colocado directamente en este curso.

Al comienzo del curso, la profesora-investigadora asignó de forma aleatoria una condición a cada uno de los dos grupos: el grupo de captación $(n=16)$ y el grupo de actividades explícitas $(\mathrm{n}=20)$.

Fueron excluidos del estudio aquellos que faltaron a más de dos sesiones de clase o aquellos miembros del grupo de actividades explícitas que no entregaron dichas actividades o que no estaban presentes el día que se corrigieron. Fueron descartados un total de 5 alumnos.

\subsection{Tratamiento y temporalización}

Para este estudio tomamos la definición de bloques léxicos de Lindstromberg y Boers (2008: 7), "secuencias de palabras que los hablantes nativos sienten como naturales y que constituyen la formulación preferida para expresar una idea o propósito" (traducción propia). La adecuación de los bloques léxicos seleccionados para su tratamiento en el aula se testó con un piloto que se administró entre hablantes nativos de español. El objetivo de este test era comprobar que la asociación entre los componentes de los bloques léxicos es efectivamente reconocible por los hablantes nativos, así que se pusieron estos bloques en enunciados y se extrajo una de las partes de cada bloque (por ejemplo: "Para las personas mayores, el mejor deporte es___ un paseo diario"). Se comprobó que los bloques previamente seleccionados eran combinaciones de palabras convencionales y generalizadas.

Los bloques léxicos meta, un total de veintiuno repartidos en tres unidades didácticas (vid. Tabla 1), estaban en relación con el tema de dicha unidad y estaban contenidos en el input al que los estudiantes estuvieron expuestos. Se trata de colocaciones léxicas prototípicas verbo- nombre (seguir consejos, dar un paseo, contraer una enfermedad) o adverbio-adjetivo (gravemente enfermo); colocaciones no prototípicas (lavar la ropa, instalar Skype, ir de excursión ${ }^{2}$ ) y otros bloques léxicos (pasarlo bien, actor de reparto). Si bien el input al que estuvieron expuestos los aprendices fue el mismo, cambió el tratamiento didáctico que se dio a dichos bloques en cada uno de los grupos.

Los dos grupos trabajaron por separado y, aunque impartidos por el mismo profesor, en cada uno se implementó una práctica pedagógica diferente en cuanto a la enseñanza de los bloques léxicos, que pasamos a detallar a continuación. En el grupo llamado de captación, se implementó un tipo de enseñanza más orientada a lo estratégico: todas las unidades meta se trabajaron en contexto, dentro del marco proporcionado por los textos. Se entrenó a los alumnos a reconocer estos bloques y se utilizaron técnicas de realce para que los captaran en el input: negritas, subrayados, preguntas del profesor y glosas con traducciones. Después de una comprensión global del significado del texto, la atención se dirigía a las formas meta, pero siempre con tareas insertadas en el marco comunicativo proporcionado por el texto. Al final de cada sesión, se pedía a los alumnos que apuntaran cinco bloques léxicos que encontraran útiles de aprender. Es decir, tal y como se ha mencionado en la introducción, en este grupo se

2 La distinción entre colocaciones léxicas prototípicas y no prototípicas es de Higueras (2006: 27). Según esta autora, las colocaciones léxicas no prototípicas están más cerca de las combinaciones libres y no cumplirían con las características de la restricción léxica, la direccionalidad y la tipicidad. 
implementaron algunos de los consejos del primer enfoque léxico (Lewis 1993, 1997, 2000), que ponía la captación en el centro de sus prácticas pedagógicas. A continuación, se puede ver un fragmento de un texto tal y como se presentó a este grupo adaptado de Corpas et al. (2005):

\section{¿A QUÉ DEDICAN LOS ESPAÑOLES SU TIEMPO LIBRE... SI LO TIENEN?}

Según un estudio reciente, solo un $13,2 \%$ de los españoles prefiere tener más horas de ocio que de trabajo. No gastan mucho dinero en viajes y prefieren pasar su tiempo libre con familiares y amigos en su propia ciudad.

En cuanto a los estudiantes en el grupo de actividades explícitas, estos estuvieron expuestos a los mismos textos que el grupo de captación (eliminando el realce de las formas meta en el caso de que lo hubiera, puesto que en el grupo de captación también se trabajó la técnica de las preguntas del profesor para dirigir la atención a los bloques léxicos). A la lectura o escucha de los textos les siguieron actividades centradas en el significado, dado que, como es ampliamente aceptado, los aprendices no pueden prestar atención al significado y a la forma al mismo tiempo, tendiendo a priorizar la comprensión del significado (Van Patten 1990; Peters 2007). Solo después de promover la comprensión del significado del texto, se hacían actividades explícitas para trabajar las unidades léxicas meta. Estas actividades que, o bien se completaron en tiempo de clase o bien en casa como deberes, eran de distinto tipo: conectar las dos partes de la colocación, insertar la colocación en un texto, personalizar la colocación, traducirla o clasificarla (ver Boers et al. 2014; Boers et al. 2016a, Pérez Serrano 2015, para una revisión).

En el siguiente cuadro, se puede ver cómo se desarrolló el estudio a lo largo de las semanas en ambos grupos:

\begin{tabular}{|l|l|l|l|}
\hline MOMENTO & NOCIONES & $\begin{array}{c}\text { MATERIALES COMUNES Y } \\
\text { ACTIVIDADES }\end{array}$ & BLOQUES LÉXICOS \\
\hline Semana 1 & $\begin{array}{l}\text { Ocio y tiempo } \\
\text { libre. }\end{array}$ & $\begin{array}{l}\text { Firma del consentimiento informa- } \\
\text { do. } \\
\text { Explicación y actividad sobre el el } \\
\text { concepto de colocación. } \\
\text { Unidad 15: Gente que se divierte. }\end{array}$ & $\begin{array}{l}\text { contar la historia } \\
\text { gastar dinero } \\
\text { pasar tiempo/ el rato } \\
\text { actor de reparto } \\
\text { tener tiempo libre } \\
\text { ir de excursión } \\
\text { pasarlo bien }\end{array}$ \\
\cline { 1 - 1 } Semana $2^{2}$ & $\begin{array}{l}\text { Unidad 15: Gente que se divierte. } \\
\text { iTe gusta el cine? }\end{array}$ \\
\hline
\end{tabular}

3 La distinción entre colocaciones léxicas prototípicas y no prototípicas es de Higueras (2006: 27). Según esta autora, las colocaciones léxicas no prototípicas están más cerca de las combinaciones libres y no cumplirían con las características de la restricción léxica, la direccionalidad y la tipicidad. 


\begin{tabular}{|c|c|c|c|}
\hline MOMENTO & NOCIONES & $\begin{array}{c}\text { MATERIALES COMUNES Y } \\
\text { ACTIVIDADES }\end{array}$ & BLOQUES LÉXICOS \\
\hline \multirow[t]{2}{*}{ Semana 3} & $\begin{array}{l}\text { Salud e } \\
\text { higiene. }\end{array}$ & $\begin{array}{l}\text { Unidad 16: Gente sana } \\
\text { Síntomas. }\end{array}$ & \multirow{2}{*}{$\begin{array}{l}\text { tomar el sol } \\
\text { seguir consejos } \\
\text { dar un paseo } \\
\text { contraer una enfermedad } \\
\text { gravemente enfermo } \\
\text { hacer dieta } \\
\text { hacerse daño }\end{array}$} \\
\hline & & $\begin{array}{l}\text { Unidad 16: Gente sana. } \\
\text { Campañas de salud. }\end{array}$ & \\
\hline \multicolumn{4}{|l|}{$\begin{array}{l}\text { Semanas } \\
4 \text { y } 5^{4}\end{array}$} \\
\hline Semana 6 & \multirow{3}{*}{$\begin{array}{l}\text { Internet. } \\
\text { Tareas de la } \\
\text { casa. } \\
\text { Objetos. }\end{array}$} & $\begin{array}{l}\text { Unidad 17: Gente innovadora. } \\
\text { Tecnología y tercera edad. }\end{array}$ & \multirow{2}{*}{$\begin{array}{l}\text { instalar Skype } \\
\text { fundirse la bombilla } \\
\text { dar órdenes } \\
\text { encender la luz } \\
\text { lavar la ropa } \\
\text { registrarse en Twitter }\end{array}$} \\
\hline Semana 7 & & Unidad 17: Gente innovadora. & \\
\hline Semana 8 & & Aplicación del cuestionario. & \\
\hline
\end{tabular}

Tabla 1. Calendario del estudio

\subsection{Instrumentos de recogida de datos}

El instrumento utilizado para este fin fueron dos cuestionarios diferentes, uno para cada grupo experimental, que se administró el último día del tratamiento didáctico en la L1 de los aprendientes. Se pidió a los alumnos que respondieran también en su lengua. La razón por la que se decidió realizar el cuestionario en inglés es que los alumnos no se sintieran coartados por un dominio insuficiente de la lengua para expresar sus valoraciones. Los cuestionarios se pueden encontrar en los apéndices I y II. El análisis se basa en las respuestas de 36 estudiantes, 16 del grupo de captación y 20 del grupo de actividades. Los cuestionarios son prácticamente iguales, excepto la primera pregunta. En ella, a los del grupo de captación se les pregunta si las rutinas para captar los bloques en el input les han funcionado para aprender vocabulario o si prefieren las actividades de léxico más tradicionales; en el grupo de actividades se indaga sobre en qué medida las actividades sobre colocaciones les han sido útiles o relevantes frente a otras actividades de vocabulario más tradicionales que han llevado a cabo en los cursos anteriores.

Asimismo, el profesor llevó a cabo un diario o bitácora en el que iba anotando todo lo concerniente a las prácticas pedagógicas diseñadas para enseñar las colocaciones meta, tanto en torno a la preparación como en torno a lo que ocurría en el aula.

4 Durante las semanas cuatro y cinco se llevaron a cabo actividades extracurriculares 


\section{ANÁLISIS Y DISCUSIÓN}

\subsection{Valoraciones de los estudiantes}

A partir de una lectura detallada de las respuestas que los estudiantes proporcionaron en los cuestionarios administrados, procedemos a un análisis basado en cuatro bloques que iremos comentando uno por uno. Entre paréntesis indicamos en qué pregunta de los cuestionarios hemos encontrado las respuestas relevantes a cada uno de los bloques.

¿Cómo perciben los estudiantes la importancia y utilidad de aprender colocaciones y otros bloques léxicos? (Pregunta número 3).

- ¿Cómo perciben los estudiantes su capacidad para captar las colocaciones en textos a los que se enfrenten en el futuro? (Pregunta número 4).

-¿Qué problemas, dificultades o inconvenientes han percibido en el proceso de enseñanzaaprendizaje de las mismas? (Preguntas número 1 y 4 ).

• ¿Cómo perciben los aprendientes la incorporación de las colocaciones a su propio discurso? (Pregunta número 5).

• ¿Cómo perciben los estudiantes la importancia y utilidad de aprender colocaciones?

La gran mayoría ve más útil centrarse en actividades que trabajan explícitamente las colocaciones: «necesitamos saber de forma explícita que ciertas palabras van juntas». Esto nos lleva a las razones que ellos han percibido acerca de por qué es útil, importante o relevante concentrarse en el aprendizaje de las colocaciones.

A continuación, recogemos las razones por las que ven útil aprender este tipo de unidades léxicas que han dado los estudiantes En primer lugar, una de las palabras más repetidas es «contexto». Los estudiantes aprecian la importancia de poner las palabras en contexto puesto que se considera que es difícil acceder a esa información de otra manera si no se trabaja en clase: «trabajar con colocaciones proporciona el contexto de cómo se usa una palabra y así no tenemos que adivinar cómo usarlas en una frase». Y ese contexto, además de permitir usarlas de forma adecuada, permite entender la función y la utilidad de las palabras.

Igual de recurrente ha sido la mención de la fluidez como razón principal por la que es útil y relevante aprender colocaciones, una fluidez que no te otorga el conocimiento de las palabras individuales: «Podrías conocer todas las palabras de una lengua y no tener fluidez en esa lengua si no conoces las colocaciones». Otros aprendientes hacen alusión también al hecho de que sin las colocaciones te podrías hacer entender sin alcanzar la fluidez deseada.

Otra de las razones más señaladas por la que los estudiantes perciben la importancia de aprender colocaciones es la de usar la lengua tal y como lo hacen los «hablantes nativos» en la «vida real». De las apreciaciones de los estudiantes se deduce que los métodos de aprendizaje de vocabulario centrados en las palabras generarían un discurso más artificial, más de aula y por consiguiente más alejado del que se genera fuera de la clase por parte de los hablantes de español: «todo el mundo puede memorizar palabras pero, ¿para qué sirve esto si no las puedes utilizar en situaciones de la vida real?». Es recurrente entender este entrenamiento en colocaciones como una suerte de aplicación de las palabras individuales a la vida real. Por tanto, de lo que se deduce de los cuestionarios, parece establecerse una asociación entre aprender palabras individuales y cumplir con los requerimientos del aula y aprender colocaciones y desenvolverte en la vida real: «las colocaciones son importantes para aprender a cómo hablar fuera de la clase, en oposición a estudiar para los exámenes del curso». 
En este sentido, se menciona su utilidad tanto para entender a los hablantes nativos en situaciones de la vida real como para producir un discurso similar al de los nativos. Sin embargo, la mayoría mencionan su utilidad en el desarrollo de actividades de la lengua productivas, a saber la expresión escrita y la oral: aprender colocaciones «hace más fácil hablar y formar frases», «te ayuda a adquirir fluidez en tu propio discurso». Es curioso cómo las han asociado más a la «lengua conversacional», repitiéndose afirmaciones como "se usan en la conversación» o «ayudan a mejorar habilidades conversacionales».

Es recurrente, asimismo, recordar la importancia y la utilidad de las colocaciones o frases por su falta de congruencia con la L1 y porque no resultan intuitivas. Los estudiantes perciben que la dificultad -y por consiguiente la necesidad de prestarles atención- de muchas de las colocaciones que se han trabajado en clase reside en la falta de una equivalencia o de una traducción literal palabra por palabra en su L1 «[aprender colocaciones] es especialmente útil cuando la colocación no se traduce literalmente». Y no solo eso, sino que además algunos perciben este aspecto como uno de los más desafiantes en el proceso de aprendizaje de una L2: «Creo que una de las partes más difíciles de una lengua es tener que descubrir todas las formas en las que la traducción directa no es posible».

También se menciona que es útil aprenderlas porque ello permite conocer el significado de las palabras que las integran: «arroja más luz sobre el significado de las palabras que otras actividades», «ayuda a aprender los matices del vocabulario mejor». Otros mencionan también la utilidad de trabajar explícitamente con colocaciones para memorizar las palabras «es más fácil memorizar el significado de una palabra cuando la has visto en contexto».

$¿$ ¿Cómo perciben los estudiantes su capacidad para captar las colocaciones en textos a los que se enfrenten en el futuro?

Algunos estudiantes admiten que han desarrollado la capacidad para captar las colocaciones durante estas semanas del curso -o que ya la tenían de antes- y que lo seguirán haciendo o que seguirán mejorando en este sentido.

Sin embargo, un grupo de la muestra, incluyendo aquí a integrantes tanto del grupo de actividades como del de captación, declara que no está seguro al respecto. Los hay que no dejan de reconocer las dificultades a la hora de captar las colocaciones en los textos: «creo que hasta cierto punto sí [que he desarrollado esa capacidad] pero creo que todavía son difíciles de descubrir para mí» o «creo que aunque soy más consciente de algunas colocaciones, es todavía muy difícil identificar las nuevas». Otros admiten su falta de habilidad y lo achacan a la falta de exposición a input «no creo que haya leído lo suficiente para captar las palabras contiguas a otras en los textos».

Por otra parte, algunos señalan cómo les resulta más fácil captar las colocaciones en los textos escritos que en los orales: «Sí, me doy cuenta de ellas en los textos, pero tengo problemas captándolas en los audios y en las películas».

¿Cómo perciben los aprendientes la incorporación de las colocaciones a su propio discurso?

Varios estudiantes han respondido que sí a esta pregunta. No obstante, un número significativo ha respondido que no o que no está seguro, y ha hecho apreciaciones bastante esclarecedoras.

Algunos aprendientes del grupo de captación admiten que «captar las frases ayuda en la comprensión de la lengua pero no tanto en su aplicación» y del grupo de tratamiento explícito recalcan: «no me siento lo suficientemente fuerte para usarlas por mí mismo». 
Otros estudiantes del grupo de captación nos recuerdan lo difícil que es llegar a usar una palabra o colocación cuando solo se ha tenido un encuentro con ella: «tengo dificultades reteniendo y aplicando estas frases que solo he oído una vez». Además, se dan cuenta de que las colocaciones a las que se presta atención son aquellas que aparecen en los textos y que no hay una aparente selección por parte del profesor: «el problema es que es imposible saber cuáles son las más importantes».

Son pocos los que especifican en qué tipo de producciones incorporan las colocaciones tratadas pero los que lo hacen mencionan más las composiciones y otras actividades de escritura. Algunos incluso mencionan la dificultad de incorporarlas al discurso oral: «creo que me resulta muy complicado, sobre todo con actividades orales».

En el grupo de tratamiento explícito se muestran algo menos escépticos ante la pregunta de si las han usado en tareas de clase, composiciones y exámenes. Son pocos los que admiten que no las han usado, justificando que «es más fácil captar que usar». Se señala que se han usado aquellas en las que se ha puesto más énfasis o «algunas de las más fáciles de recordar» admitiendo que «otras son más difíciles para mí porque no parece que las palabras se relacionen».

Un estudiante señala los beneficios de realmente memorizar una colocación: «una vez que aprendo la colocación, suena natural y me libera de componer una frase en inglés y traducirla en mi cabeza».

¿Qué problemas, dificultades o inconvenientes han percibido en el proceso?

En el grupo de realización de actividades, una respuesta frecuente ante la pregunta de qué les funciona mejor, si hacer actividades centrándose en las colocaciones o en las palabras individuales, es que para ellos sería útil una combinación de los dos tipos de actividades. En muchos casos responde a la creencia de que cuanto más se practiquen las formas meta y de diversas formas, mejor se aprenderán. Otra razón que aducen de forma recurrente es que con las actividades tradicionales de vocabulario adquieren una mayor cantidad de palabras y que esto los ayuda también porque al hablar sienten limitaciones por no conocer suficientes palabras.

En los casos en los que prefieren actividades que se centren en el desarrollo del conocimiento de palabras individuales aducen razones de currículo y evaluación: «creo que [aprender colocaciones] ayuda mucho con la fluidez pero cuando estudié para el examen me di cuenta de que había muchas palabras individuales que no sabía».

En cuanto al grupo de captación de las formas meta, las dificultades señaladas son mayores. Ante la pregunta de si prefieren captar las colocaciones en los textos en lugar de hacer actividades que se centren en palabras individuales los problemas señalados versan en torno a tres ejes que detallamos a continuación.

En primer lugar, hemos encontrado afirmaciones que revelan las creencias de los aprendientes y sus hábitos de aprendizaje. A este respecto, es frecuente encontrar afirmaciones como «es más fácil aprender con actividades». Hacer una actividad con un objetivo específico ofrece un resultado tangible y esto proporciona más seguridad en cuanto a que pueden ver de forma más plausible cómo está evolucionando en el aprendizaje. Algunos estudiantes han percibido como insuficiente una metodología que promueve un aprendizaje semiimplícito de las colocaciones: «creo que captar las colocaciones es una forma muy buena de aprender vocabulario para los niños, pero dado que para los adultos es más difícil aprender de forma innata, creo que las actividades son muy importantes». 
En segundo lugar, es muy frecuente aludir al problema de la memoria. Varios estudiantes del grupo experimental de captación de la forma meta han percibido que captar las colocaciones no les ayuda a memorizarlas: «creo que percibir las colocaciones en el input es útil pero personalmente, no me ayuda a recordar el vocabulario». Algunos mencionan además que para memorizar palabras necesitan hacer ejercicios de drill.

Por último, también han señalado en varias ocasiones la ya mencionada cuestión del examen y las exigencias del currículo: «es muy importante enumerar y hacer más explícito qué tenemos que saber» o «creo que las actividades de palabras individuales son muy útiles para el examen parcial y final, especialmente para la sección de selección múltiple». Estos comentarios revelan, efectivamente, una cierta falta de congruencia entre la metodología empleada en el aula para aprender vocabulario prestando atención al eje sintagmático y la evaluación de los ítems de vocabulario en los exámenes administrados. Aun siendo conscientes de ello, no nos fue posible elaborar una evaluación más acorde con la metodología, puesto que los exámenes son comunes a todo el programa.

\subsection{Valoraciones del profesor}

A lo largo del estudio experimental, el profesor-investigador recogió algunas de sus impresiones en un diario. Recogemos a continuación aquellas reflexiones que nos parecen más relevantes.

- Creo que el entrenamiento en la tarea de captación requiere cierta práctica por parte del profesor. Al comienzo del estudio, pedía a los estudiantes participación en grupo clase para que ellos mismos contribuyeran en la labor de localizar las colocaciones de un texto. No lograba con esta dinámica la participación y las aportaciones que yo estaba buscando, así que me fui dando cuenta de que para lograr una mayor fluidez a la hora de trabajar con las colocaciones de un texto, tenía que dirigir de forma más sistemática esa captación y con distintas técnicas para no hacerlo repetitivo: el realce en el propio texto a través de negritas o subrayados o la búsqueda de colocaciones a partir de sinónimos o de sus equivalentes en inglés. Sin embargo, la realización de actividades más dirigidas que se realizó en el otro grupo me dio una mayor sensación de seguridad porque fue más fácil implicar al estudiante en este tipo de tareas.

- Tanto en la realización de las actividades como en la captación de las colocaciones en el input, me ha dado la impresión en alguna ocasión de que, al no tratarse de palabras «difíciles» las colocaciones meta les resultaban fáciles y obvias ya que la mayoría de ellas no planteaban ningún problema de comprensión. Transmitir la idea de que es bueno dedicarle tiempo de aula a este tipo de ítems lleva tiempo. Me he dado cuenta de que para implementar algunas de las prácticas de un enfoque léxico también es bueno trabajar con las creencias de los estudiantes.

- He notado que estudiantes de ambos grupos, aunque especialmente el grupo de captación, me empezaban a hacer preguntas de motu proprio acerca de algunas colocaciones y cómo ellos se van dando cuenta de que la traducción palabra por palabra no funciona con las colocaciones. Sirva como ejemplo la reproducción de esta conversación entre dos estudiantes a propósito de dejar de fumar:

(Alumno 1): Pero, dejar es to leave, ¿no?

(Alumno 2): Pero aquí no significa eso, ¿no? 
- He notado también uno de los problemas que presenta la aplicación de un enfoque léxico en cuanto al español, una lengua con una flexión rica. Lo explicaré con un ejemplo. Hablando de la película Eva, en el input, aparecía La dirige Kike Maillo. Muchos estudiantes han interpretado la como artículo y dirige como sustantivo, de tal forma que su producción era la dirige es... (como equivalente a el/la director/a es...). Este error fue frecuente entre los estudiantes de ambos grupos y está derivado de promover una enseñanza en bloques no analizados.

- Por lo demás, he comprobado que hay que animarlos, a la hora de producir, a utilizar las colocaciones que aparecen en el input, porque si no van a las estructuras que ya saben y «dominan». Por ejemplo: «La película es en NY». Hay que animarles a utilizar un léxico que aparecía en el input «La película está ambientada en NY». Es decir, la tarea no se queda en promover su reconocimiento en los textos y hacer actividades para su memorización. Se han de proporcionar oportunidades para la incorporación de las mismas en el propio discurso de los estudiantes, guiándolos, proponiéndoles alternativas léxicas, etc.

\section{CONCLUSIONES E IMPLICACIONES PEDAGÓGICAS}

Los datos obtenidos a través de este análisis nos permiten afirmar que los estudiantes no cuestionan la importancia de aprender colocaciones léxicas y otras unidades léxicas complejas por razones como la utilidad de cara al uso de las palabras en contexto, la mejora de la fluidez, el acercamiento al discurso de los nativos, la posibilidad de desenvolverse en situaciones de comunicación reales o porque ayuda a aprender los matices de las palabras individuales. Sin embargo, consideran que es necesario también aprender palabras individuales por razones diversas, como aumentar el número de palabras conocidas o enfrentarse mejor preparados a las exigencias del examen. Esto nos lleva a reflexionar sobre el alcance de la aplicación de un enfoque léxico, que no debe quedarse en la selección de materiales y actividades sino que debe llegar también a la evaluación de la misma competencia fraseológica y colocacional que se pretende desarrollar en los aprendices.

Por otro lado, y ya en cuanto a la instrucción, pese a que algunos aprendices sí consideran que han adquirido el hábito de captar y reconocer las colocaciones en los textos a los que se enfrentan y se ven capaces de hacerlo en el futuro, otros ven difícil ponerlo en práctica de forma autónoma, sobre todo en los textos orales. Es decir, tanto los datos cuantitativos obtenidos en otros estudios (Peters 2012; Boers et al. 2016a) como las propias impresiones de los estudiantes aquí recogidas parecen indicar que el entrenamiento en la captación de los bloques difícilmente se hará de forma autónoma e independiente.

Las apreciaciones más esclarecedoras en torno a su futuro uso de las colocaciones y otros bloques aprendidos en clase las han hecho algunos integrantes del grupo de captación. Desde una perspectiva cuantitativa ya se ha demostrado que esta técnica es menos eficaz que las actividades explícitas (Szoudarsky 2012; Pérez Serrano 2018): hacer actividades explícitas genera un mayor aprendizaje que captarlas en el input. Vemos aquí que, en consonancia con lo que se afirma en la literatura anterior (Boers y Lindstromberg 2009), los estudiantes aprecian que la captación ayuda en la comprensión de la lengua pero no promueve la incorporación al propio discurso: esta práctica pedagógica no les da seguridad y sienten que no les ayuda a recordar: se sienten más seguros con la realización de actividades. En consecuencia, nos aventuramos a afirmar que podemos confiar en las técnicas de captación para un primer acercamiento a los bloques léxicos o para una consolidación de aquellos que 
se conocen parcialmente y siempre con un trabajo de cara a las creencias de los estudiantes, concienciándolos de la utilidad de este tipo de prácticas.

Así, si bien la práctica de las actividades resulta más de la preferencia de los estudiantes y les dan más seguridad de cara a la incorporación de las unidades léxicas meta en su propio discurso, no debemos denostar las técnicas de captación. Estas, es cierto, generan más incomprensión por parte del estudiante, pero dada la cantidad de bloques léxicos que puebla el discurso, no podemos pretender enseñarlos todos de forma explícita en el aula. La captación genera ganancias de aprendizaje (Peters 2012; Szudarsky y Carter 2016; Boers et al. 2016a) y consume menos tiempo y esfuerzo. A raíz de las preguntas a los estudiantes, nos damos cuenta de que la implementación de este tipo de práctica conlleva necesariamente una labor de concienciación y de trabajo de las creencias de los alumnos por parte del profesor. Una combinación de ambas técnicas resultaría adecuada, dejando las técnicas de captación para aquellas colocaciones o bloques que ya se conocen o que son congruentes con la L1 del estudiante y hacer actividades explícitas sobre aquellos que ofrecen más dificultad (por ejemplo, los que no son congruentes con la L1 del estudiante, los que se encuentran en bandas de frecuencia medias o los que están formados por palabras con poco contenido léxico como hacer, tomar, etc.).

\section{REFERENCIAS BIBLIOGRÁFICAS}

Barfield, A. (2006). An Exploration of Second Language Collocation Knowledge and Development. Tesis no publicada, University of Wales: Swansea.

Boers, F., J. Eyckmans, J. Cappel, H. Stengers, M. Demecheleer (2006). "Formulaic sequences and perceived oral proficiency: putting a Lexical Approach to the test", Language Teaching Research, 10, pp. 245- 261. DOI: https://doi.org/10.1191/13621688061r195oa

Boers, Frank y S. Lindstromberg (2009). Optimizing a lexical approach to instructed second language acquisition, Basingstoke: Palgrave Macmillan.

Boers, Frank, M. Demecheleer, A. Coxhead y S. Webb (2014). "Gauging the effects of exercises on verb-noun collocations", Language Teaching Research, 0 (0), pp. 1-21. DOI: https://doi. org $/ 10.1177 / 1362168813505389$

Boers, F., M. Demecheleer, L. He, J. Deconinck, H. Stengers y J. Eyckmans (2016a). "Typographical enhancement of multiword units in second language texts", International Journal of Applied Linguistics, 27, 2, pp. 448-469. DOI: https://doi.org/10.1111/ijal.12141

Boers, Frank, T. Dang y B. Strong (2016b). "Comparing the effectiveness of phrase-focused exercises: A partial replication of Boers, Demecheleer, Coxhead, and Webb (2014)", Language Teaching Research, 21, 3, pp. 362 - 380. DOI: https://doi.org/10.1177/1362168816651464

Corpas, J., A. Garmendia y C. Soriano (2005). Aula Internacional 2. Curso de español. Barcelona: Difusión.

LE-THI, D., M. P. Rodgers y A. Pellicer-Sánchez (2018). “Teaching Formulaic Sequences in an English- Language Class: The Effects of Explicit Instruction Versus Coursebook Instruction”, TESL Canada Journal, 34(3), pp. 111-139. DOI: https://doi.org/10.18806/tesl.v34i3.1276

Higueras, M. (2006). Las colocaciones en la clase de ELE. Madrid: Arco Libros.

LewIs, M. (1993). The Lexical Approach, Londres: Language Teaching Publications.

LewIs, M. (1997). Implementing the Lexical Approach, Londres: Language Teaching Publications.

Lewis, M. (2000). Teaching collocation. Further Developments in the Lexical Approach, Londres: Language Teaching Publications.

LI, J. y N. Schmitt (2010). "The development of collocation use in academic texts by advanced L2 learners: A multiple case study approach". En Wood, D. (ed.). Perspectives on formulaic language: Acquisition and communication, Nueva York: Continuum, pp. 22-46. 
Lindstromberg, S. y F. Boers. (2008), Teaching Chunks of Language. From Noticing to Remembering. Londres: Helbling Languages.

Nekrasova, T. (2009). "English L1 and L2 speakers' knowledge of lexical bundles", Language Learning, 59, pp. 647-686. DOI: https://doi.org/10.1111/j.1467-9922.2009.00520.x

Pérez Serrano, M. (2015). Un enfoque léxico a prueba: efectos de la instrucción en el aprendizaje de las colocaciones léxicas. Tesis doctoral inédita.

Pérez Serrano, M. (2018). "Which type of instruction fosters chunk learning? Preliminary conclusions”, Revista de lingüística y lenguas aplicadas, 13, pp. 133-143. DOI: https://doi.org/10.4995/ rlyla.2018.7886

Peters, E. (2007), "The influence of Task Instruction on Vocabulary Acquisition and Reading Comprehension”. En García Mayo, M.P. (ed.). Investigating Task in Formal Language Learning. Clevedon: Multilingual Matters, pp. 178-98.

Peters, E. (2012). "Learning German formulaic sequences: The effect of two attention- drawing techniques", Language Learning Journal, 40, pp. 65-79. DOI: https://doi.org/10.1080/0957173 6.2012 .658224

Sinclair, J. (1991). Corpus, concordance, collocation: Describing English language. Oxford: Oxford University Press.

Schmitт, N. y F.A. Alali (2012). "Teaching Formulaic Sequences: the same as or different from teaching single words?", TESOL Journal, 3.2, pp. 153-180. DOI: https://doi.org/10.1002/tesj.13

Stengers, H. (2009). Putting the idiom principle to the test: An exercise in applied comparative linguistics. Tesis doctoral inédita, Universidad de Bruselas.

Stengers, H. y F. Boers (2015). "Exercises on collocations: a comparison of trial-and-error and exemplar-guided procedures", Journal of Spanish Language Teaching, 2-2, pp. 152-164. DOI: ttps://doi.org/10.1080/23247797.2015.1104030

SzUDARSKI, P. (2012). "Effects of meaning-and form-focused instruction on the acquisition of verbnoun collocations in L2 English", Journal of Second Language Teaching \& Research, 1(2), pp. $3-37$.

SzudARski, P. y R. Carter (2016). "The role of input flood and input enhancement in EFL learners' acquisition of collocations", International Journal of Applied Linguistics, 26, pp. 245-265. DOI: https://doi.org/10.1111/ijal.12092

VanPatten, B. 1990. "Attending to form and content in the input: an experiment in consciousness", Studies in Second Language Acquisition, 12, pp. 287-301. DOI: https://doi.org/10.1017/ S0272263100009177

Wray, A. (2002). Formulaic language and the lexicon, Cambridge: Cambridge University Press.

YANG, Y. y A. Hendricks (2004). "Collocation awareness in the writing process", Journal of Reflections on English Language Teaching, 3, pp. 51-78.

YING, Y., y M. O'Neill (2009). "Collocation Learning through an "AWARE” Approach: Learner Perspectives and Learning Process". En A. Barfield y H. Gyllstad (eds.). Researching Collocations in Another Language: multiple interpretations, Basingstoke, Palgrave Macmillan, pp. 181-193.

YAMASHITA, J. Y N. Jiang (2010). "L1 influence on the acquisition of L2 collocations: Japanese ESL users and EFL learners acquiring English collocations", TESOL Quarterly, 44, pp. 647-668. DOI: https://doi.org/10.5054/tq.2010.235998 


\section{ApÉNDiCe I: CUESTIONARIO PARA EL GRUPO DE CAPTACIÓN}

\section{Cuestionario sobre tu aprendizaje de vocabulario}

During these past weeks, you have hardly done specific activities to learn words. But we have actually worked with vocabulary: what we have done to learn new vocabulary is noticing collocations and expressions in the texts/audios and we have underlined and/or noting them down.

1) Do you think that noticing collocations and expressions is a good way of learning vocabulary or do you think doing activities to learn single words works better for you (as in, for example, the vocabulary activities in My Spanish Lab such as synonyms and antonyms, cross the odd one out, etc.)?

2) Had you previously heard about collocations? Are you aware of having worked with them in other Spanish/foreign language classrooms?

3) Why do you think it is useful/important/relevant to focus on collocations rather than single words? (For example, instead of learning just the word sol, learn it in its collocation tomar el sol.)

4) Do you think that after these weeks you have acquired the habit of noticing not only the new words but also its contiguous words in the texts? If so, do you think you are going to keep on doing it whenever you confront texts (writings, audios, movies, etc.) on your own?

5) Are you aware of having used the collocations/expressions that your instructor has annotated on the board in compositions, classroom activities (both oral and written) and in the exam (collocations such as tener la tensión alta, ir de tapas, fundirse una bombilla)? 


\section{ApÉNDiCE II: CuestionaRio PARA EL GRUPO DE TRATAMIENTO EXPLí́cITO}

\section{Cuestionario sobre tu aprendizaje de vocabulario}

During these past weeks, you have hardly done specific activities to learn single words. Instead, we have made activities to learn collocations and phrases relevant to each topic (leisure, health, technology).

1) Do you think that focusing on collocations and expressions is a good way to learn vocabulary or do you think doing activities to learn words works better for you (as in, for example, vocabulary activities in My Spanish Lab such as synonyms and antonyms, cross the odd one out, etc.)?

2) Had you previously heard about collocations? Are you aware of having worked with them in other Spanish/ foreign language classrooms?

3) Why do you think it is useful/ important/ relevant to focus on collocations rather than single words? For example, instead of learning just the word sol, learn it in its collocation tomar el sol.

4) Do you think that after these weeks you have acquired the habit of noticing not only the new words but also its contiguous words in the texts you read or listen to? If so, do you think you are going to keep on doing it whenever you confront texts (also audios and movies) on your own?

5) Are you aware of having used the collocations/ expressions that your instructor has annotated on the board in compositions, classroom activities (both oral and written) and in the exam (collocations such as tener la tensión alta, ir de tapas, descargarse música)? 